\title{
Clinical profile and outcome of dengue patients with atypical features in the pediatric age group in a tertiary care center of East India
}

\author{
Sumita Pal', Basudha Roy ${ }^{2}$, Amrit Chattopadhyay ${ }^{3}$, Akhila Andra ${ }^{4}$, Gautam De ${ }^{5}$ \\ ${ }^{1}$ Associate Professor, ${ }^{2,4}$ Resident, ${ }^{5}$ Professor, Department of Pediatrics, Calcutta National Medical College, ${ }^{3}$ Resident, \\ Department of Neuromedicine, Medical College, Kolkata, West Bengal, India
}

Background: Dengue is one of the most widespread arthropod borne diseases worldwide with an annual incidence of 50-100 million cases per year. The recent resurgence of dengue in the pediatric population has created concern among pediatricians, especially due to increased incidence of atypical features, leading to delayed diagnosis and increased mortality. There are few studies regarding this due to under reporting of the cases. Aims and Objectives: This study aims to study the clinical profile and outcome of dengue patients between the age groups of 1 month and 12 years with special emphasis on patients with atypical features. Dengue patients with atypical presentation are easily misdiagnosed by physicians, especially in the pediatric age group which increases the morbidity and mortality. Materials and Methods: This was a retrospective observational study conducted from May 2018 to September 2019 in the Department of Pediatrics, CNMCH. All pediatric patients who were dengue IgM or dengue NS1 positive were included in the study and their course of stay was followed through hospital records. The data were statistically analyzed through SPSS 16.0 software. Results: Sixtytwo dengue patients were included in the study. Thirty-two patients presented as mild febrile illness, classical dengue fever (DF), dengue hemorrhagic fever, or shock syndrome without atypical manifestations. Atypical manifestations were found in 30 patients $(48.38 \%)$ of our study. Eleven (17.7\%) patients had splenomegaly, $5(8.1 \%)$ patients had hepatitis, $3(4.8 \%)$ patients had diarrhea, $2(3.2 \%)$ patients had acalculous cholecystitis, 1 (1.6\%) patient had hepatitis with acalculous cholecystitis, $1(1.6 \%)$ patient had hepatitis with diarrhea, 1 (1.6\%) patient had myocarditis, $1(1.6 \%)$ patient had paroxysmal supraventricular tachycardia, $1(1.6 \%)$ patient had acute pancreatitis, $1(1.6 \%)$ patient had acute kidney injury, $1(1.6 \%)$ patient had ARDS, $1(1.6 \%)$ patient had encephalitis, and $1(1.6 \%)$ patient had myositis. Need of intensive care facility was more in patients with atypical manifestations due to mis or late diagnosis which prolonged course of stay. Conclusion: Atypical manifestations of DF are more common than reported. Lack of awareness among physicians, especially in primary health center, leads to delayed diagnosis and increased mortality and morbidity.

Key words: Atypical feature; Case fatality; Dengue fever; Early disease detection

\section{Access this article online}

\section{Website:}

http://nepjol.info/index.php/AJMS DOI: 10.3126/ajms.v12i11.3929

E-ISSN: 2091-0576

P-ISSN: 2467-9100

Copyright (c) 2021 Asian Journal of Medical Sciences

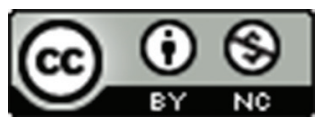

This work is licensed under a Creative Commons Attribution-NonCommercial 4.0 International License.

\section{INTRODUCTION}

Dengue viral infection has grown dramatically around the world in recent decades. It has emerged as a serious international public health threat with almost half of the world's population at risk creating a sense of utmost fear in general population. Although the full global burden of the disease is uncertain, there are estimated 50-100 million cases every year with 500,000 people requiring hospitalisation annually. About $2.5 \%$ of the patients die. ${ }^{1}$ 
The resurgence of dengue has led to frequent outbreaks in both urban and rural parts of the country. Interactions between crucial factors such as environment, vector, virus, and ecology play an important role in dengue outbreaks. ${ }^{2}$ Dengue presents with a range of clinical symptoms often with unpredictable clinical evolution and outcome. Infection by any of the four dengue virus serotypes may be asymptomatic or may lead to classic dengue fever (DF) or more severe forms of the disease, namely, dengue hemorrhagic fever (DHF) and dengue shock syndrome (DSS).

The 2009 WHO revised criteria classify dengue virus infection into dengue with or without warning signs and severe dengue (dengue with severe plasma leakage, severe bleeding, or multiorgan failure). ${ }^{2} \mathrm{~A}$ wide spectrum of unusual manifestations of dengue affecting various organ systems have recently become more frequent, which might be under-reported because of lack of awareness. There are very few studies, which have documented unusual clinical profile of dengue.

Globally, 50 million dengue infections are reported annually. ${ }^{1}$ In India, the annual incidence is estimated to be 7.5-32.5 million. ${ }^{3}$ In Odisha, a state of East India, the first outbreak was reported in 2010, followed by extensive outbreaks in 2011, affecting a large number of people. Accordingly to $\mathrm{WHO}$, the case fatality rate for dengue is roughly $5 \%$. Aedes albopictus was found to be the most abundant vector in the areas surveyed in Orissa followed by Aedes aegypti. DENV-2 was the prevailing serotype. ${ }^{4}$

The case fatality rate in patients with severe dengue infection which consists of DHF and DSS can be as high as $44 \% 0^{2,5}$

The incubation period after the mosquito bite is 3-8 days. Infants and young children often have a non-specific febrile illness that can hardly be differentiated from other viral illnesses. The more severe cases of DF are usually seen in older children and are characterized by a rapidly rising temperature $\left(\geq 39^{\circ} \mathrm{C}\right)$ that lasts 5-6 days, sometimes returning to almost normal in the middle of the febrile period (biphasic or saddleback temperature curve). The febrile period is accompanied by severe headache, retroorbital pain, myalgia, arthralgia, nausea, and vomiting. Over half of infected people report a rash during the febrile period that is initially macular or maculopapular and becomes diffusely erythematous, sparing small areas of normal skin ("islands of white in a sea of red"). ${ }^{6}$ Minor hemorrhagic manifestations such as petechiae, epistaxis, and gingival bleeding do occur. Severe hemorrhage is unusual. DF may be very incapacitating, but its prognosis is favorable and recovery generally occurs after 7-10 days of illness. In Asia, DHF and DSS mainly affect children under 15 years of age in hyperendemic areas. ${ }^{7}$

An increasing number of dengue infections have been related to other unusual manifestations. These include DF with severe hemorrhage, fulminant liver failure, cardiomyopathy, and neurological phenomena such as altered consciousness, convulsions, and coma resulting from encephalitis and encephalopathy. ${ }^{8}$

\section{MATERIALS AND METHODS}

This was a retrospective observational study conducted from May 2018 to September 2019 in the Department of Pediatrics, $\mathrm{CNMCH}$. The study was preapproved by Institutional Ethical Committee. All pediatric patients who were dengue IgM and/or dengue NS1 positive were included in the study and their course of stay was followed through hospital records. The laboratory investigations such as complete blood count, liver function test, renal function test, cerebrospinal fluid examination, chest X-ray, and ultrasonography were analyzed.

\section{Control}

Not required.

\section{Inclusion criteria}

A) Patients aged in between 1 month and 12 years.

B) Dengue NS1 and/or IgM positive.

\section{Exclusion criteria}

A) Patients aged $<1$ month and more than 12 years.

B) Any known diagnosed case of fever other than dengue.

C) Any fever that is neither NS1 nor IgM positive.

\section{Study variables}

Demographics, clinical features, and laboratory parameters.

\section{Statistical analysis}

Descriptive statistics were expressed as number and percentages. Data were analyzed using SPSS software. $\mathrm{P}=0.05$ or less was considered as statistically significant.

\section{RESULTS}

Sixty-two pediatric patients aged 1 month-12 years who were dengue NS1 or dengue IgM positive, admitted in the Department of Paediatrics, Calcutta National Medical College, Kolkata, during the period of May 2018-September 2019 were included in the study. Thirty-two patients did not present with atypical features. Seventeen $(27.4 \%)$ patients had classical DF, $8(12.9 \%)$ patients had dengue hemorrhagic fever, and $7(11.3 \%)$ patients had dengue 
shock syndrome. Atypical manifestations were found in 30 patients $(48.38 \%)$.

According to atypical manifestation, 11 (17.7\%) patients had splenomegaly, and in fact, it was the most common atypical manifestation of our study. Among the atypical gastrointestinal manifestation, we found that $5(8.1 \%)$ patients had hepatitis, $1(1.6 \%)$ patient had hepatitis with acalculous cholecystitis, $1(1.6 \%)$ patient had hepatitis with diarrhea, $2(3.2 \%)$ patients had only acalculous cholecystitis, and $1(1.6 \%)$ patient had acute pancreatitis. Three $(4.8 \%)$ patients presented as cases of febrile diarrhea. Patients with hepatitis were corroborated with laboratory investigations and showed deranged liver enzymes. The patient presenting as acute pancreatitis had raised serum amylase and lipase and USG whole abdomen showing hypoechoic peripancreatic areas of inflammation. The patient responded to symptomatic treatment.

Clinically, we found that $11(17.7 \%)$ patients had bleeding manifestation among which $1(1.6 \%)$ patient had local bleeding from mouth and gum, $2(3.2 \%)$ patients had hematemesis, and $2(3.2 \%)$ patients had hematuria. Most of them showed low platelet count.

Three (4.8\%) patients had dizziness, $3(4.8 \%)$ patients had less frequency of micturition, $4(6.5 \%)$ had loose stools, 1 $(1.6 \%)$ had palpitation and sweating, $14(22.6 \%)$ patient had altered sensorium and $1(1.6 \%)$ patient had convulsion, $37(59.7 \%)$ patients had pallor, $3(4.8 \%)$ patients had icterus, $9(14.5 \%)$ patients had edema, and $11(17.7 \%)$ patients had lymphadenopathy.

We found one patient with neurological presentation in the form of acute encephalitis syndrome. The patient presented with generalized tonic-clonic seizure. The patient needed anticonvulsant therapy for the management of convulsions. MRI brain and EEG done to rule out pre-existing neurological problem were normal.

The cardiological complications encountered were acute myocarditis and conduction abnormality in the form of paroxysmal supraventricular tachycardia (PSVT). The patient with myocarditis had cardiomegaly in echo cardiography and digital chest X-ray. ECG showed sinus tachycardia, non-specific ST-segment and T-wave abnormalities, and patient needed ionotropic support. PSVT was diagnosed by ECG and was treated by adenosine. Both of these patients had long hospital stay but recovered completely.

ARDS is one of the unusual and fatal complications of severe dengue infection. In our study, one patient had severe respiratory distress and chest X-ray showing diffuse groundglass opacity and consolidation suggestive of ARDS. The patient needed non-invasive ventilation support.

Acute kidney injury was present in one case of our study. The patient had raised urea and creatinine with oliguria. The patient responded very well to monitored fluid and serum electrolytes management.

Even if myalgia is a common feature of dengue, myositis is uncommon and an atypical manifestation. We had a case of myositis, with very high serum CPK value $(>1000 \mathrm{mcg} / \mathrm{L})$. Thus in children presenting with myositis, possibility of dengue should be kept in mind (Figure 1).

Among 62 dengue patients, $24(38.7 \%)$ patients had to be shifted to PICU out of which 16 (25.8\%) had atypical features. All of the patients were cured and discharged from hospital. There was no mortality. Need for PICU admission depended on factors such as impending decompensated

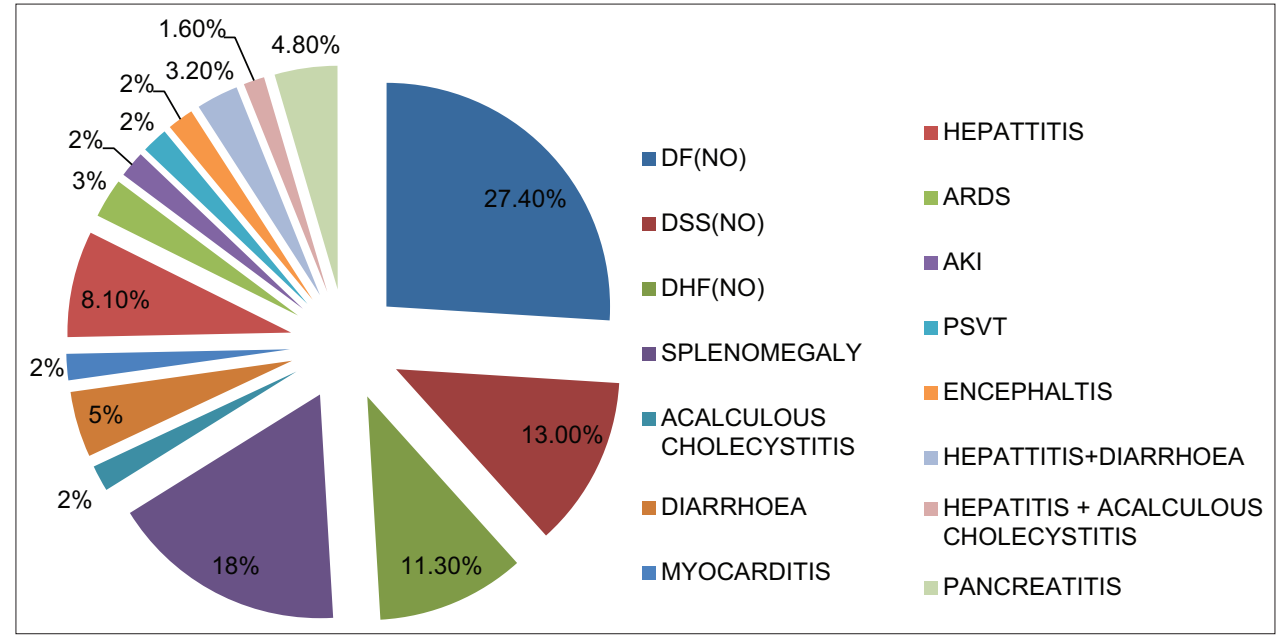

Figure 1: Pie chart showing various clinical manifestations of the patients along with atypical features 
shock, severe respiratory distress, altered sensorium, and need for cardiac monitoring. Increased mean hospital stay was observed in patients of ARDS, myocarditis, PSVT, encephalitis, and acute kidney injury.

The mean duration fever (mean \pm s.d.) of patients was 6.16 \pm 2.00 days. The mean hospital stay (mean \pm s.d.) of patients was $8.22 \pm 4.68$ days. Difference of mean hospital stay days in patients without versus with atypical manifestation was statistically significant $(<0.0001)$ [Table 1].

Association of atypical manifestation versus PICU admission was statistically significant $(<0.0001)$.

\section{DISCUSSION}

The most common atypical feature in our study was organomegaly in the form of splenomegaly. Splenomegaly and lymphadenopathy signified the occurrence of dengue virus antigen in the lymphoreticular cells.

Among the systems involved, the most common was the gastrointestinal system. Five (8.1\%) patients had hepatitis. The etiology of hepatic dysfunction in DF is usually due to direct cytopathic injury, unregulated host immune response, active viral replication, and hypoxia with tissue ischemia due to prolonged shock, hemorrhage, and metabolic acidosis.

Acute pancreatitis found in one patient is a rare complication of DF. It is possibly due to direct cytopathic effect of virus or an autoimmune response by molecular mimicry causing pancreatic outflow obstruction by resulting edema and ultrasound abdomen is characterized by enlarged pancreas and elevated serum amylase and lipase levels.' Acute acalculous cholecystitis is equally rare in DF. The main pathophysiological changes in DF could be due to increased vascular permeability causing plasma leakage and serous effusion with high-protein content which causes thickening of gallbladder wall. ${ }^{10}$ The course of the disease is usually self-limiting and gallbladder wall thickness usually returns to normal with supportive care in majority of cases, even though isolated cases of gangrene and perforated gallbladder with peritonitis have been reported and surgical intervention is reserved only for children who have evidence of gangrene, perforation, and diffuse peritonitis. Acute acalculous cholecystitis was seen in two children who improved symptomatically and were discharged.

Acute kidney injury was present in one patient in the present study. It is an unusual manifestation of DF and mainly presents as shock-induced acute tubular necrosis apart from other rare causes such as multiorgan dysfunction and rhabdomyolysis. The role of immune complex in the development of renal failure in dengue infection is still unclear.

Although myalgia is a common manifestation of DF, myositis is unusual but was found in one of our cases. The probable mechanism for myositis is the release of myotoxic cytokines, particularly tumor necrosis factor-alfa, thereby injuring the affected muscle.

We found that the mean age (mean \pm s.d.) of patients was $59.65 \pm 37.73$ months (around 6 years). We also found that $34(54.8 \%)$ patients were female and $28(45.2 \%)$ patients were male. According to residential status, 36 (58.1\%) patients were rural and $26(41.9 \%)$ patients were urban. In his study, Pothapregada et al., ${ }^{11}$ found that the most common affected age groups was $6-12$ years $(58.2 \%)$ and the mean age of presentation was 6.9 (3.0) years. Maleto-female ratio was 1.2:1. Pothapregada et al., ${ }^{11}$ found that fever duration of $<7$ days was present in 185 cases

Table 1: Difference of mean hospital stay days in patients without versus with atypical manifestation

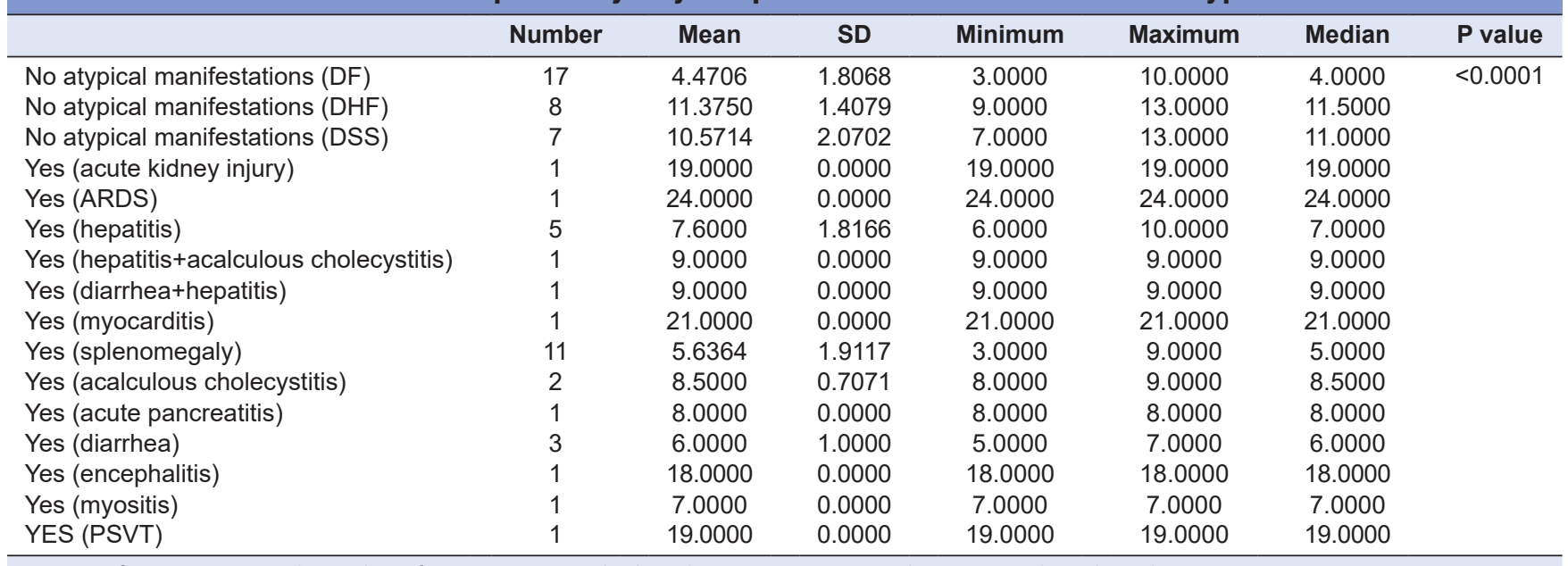

DF: Dengue fever, DHF: Dengue hemorrhagic fever, DSS: Dengue shock syndrome, PVST: Paroxysmal supraventricular tachycardia 
(72.8\%), >7 days in 23 cases ( $9 \%)$, and biphasic fever in 46 cases $(18.1 \%)$. The most common clinical warning signs at admission were persistent vomiting $(77.1 \%)$, liver enlargement $(62.9 \%)$, cold and clammy extremities $(42.5 \%)$, pain abdomen $(31.8 \%)$, hypotension $(31.4 \%)$, restlessness $(27.1 \%)$, giddiness $(23.6 \%)$, bleeding $(20.1 \%)$, oliguria $(12.9 \%)$, lethargy $(6.3 \%)$, pleural effusion $(5.1 \%)$, and impaired consciousness with Glasgow Coma Scale $<8(1.9 \%)$.

We found that the mean duration fever (mean \pm s.d.) of patients was $6.16 \pm 2$ days, $47(75.8 \%)$ patients had body ache, 44 (71.0\%) patients had vomiting, 40 (64.5\%) patients had abdominal pain, and $35(56.5 \%)$ patients had had rash. After fever, body ache was found to be the most consistent symptom followed by vomiting and pain abdomen.

Our study found that $11(17.7 \%)$ patients had bleeding manifestation. One $(1.6 \%)$ patient had local bleeding from mouth and gum, 2 (3.2\%) patients had hematemesis, and $2(3.2 \%)$ patients had hematuria. The bleeding manifestations also did not correlate well with platelet counts and occurred in children with even normal platelet counts and platelet transfusion was given in children with severe dengue with severe thrombocytopenia. In his study, Sreenivasa et al., ${ }^{12}$ studied the common bleeding manifestations in dengue. A total of 100 children with a mean age of $7.9 \pm 3.7$ years were included in his study. During the course of illness, bleeding occurred in $60 \%$ of children. It was found that $26 \%$ of children had melena,

$20 \%$ had petechiae, $8 \%$ had hematemesis, $4 \%$ had epistaxis, and $2 \%$ had gum bleeding. All $(100 \%)$ the patients who had platelet counts between 50,001 and 100,000/cu.mm developed hemorrhage and $95.8 \%$ of patients with platelet range between 20,001 and 50,000/cu.mm of blood had developed hemorrhage.

Kakoti et al, ${ }^{13}$ found that recent studies have shown dengue viral infection causing encephalitis with high morbidity and mortality. In our study, one patient presented with encephalitis and needed PICU support and anticonvulsant therapy.

\section{Limitations of the study}

In spite of every sincere effort, my study has lacunae.

The notable short comings of this study are as follows:

1. The sample size was very small. Only 62 cases are not sufficient for this kind of study. It may produce a wrong perception of outcome.

2. The study has been done in a single center.

3. The study was carried out in a tertiary care hospital, so hospital bias cannot be ruled out.

\section{CONCLUSION}

Atypical manifestations of dengue can be present along with the classical manifestations and are more common than actually reported. They may be the only presentation as well. Thus, it is essential for treating physicians to be aware of these deceiving presentations of dengue infection for early diagnosis and for better treatment of patients. This will cause significant decrease in case fatality rate of dengue.

\section{ACKNOWLEDGMENT}

We express our heartfelt thanks to our patients and their attendants for their cooperation and for keeping faith in us. We are also grateful to our head of department, other faculty members and students, for the constant support and encouragement.

\section{REFERENCES}

1. Rigau-Perez JG, Clark GG, Gubler DJ, Reiter P, Sanders EJ and Vorndam AV. Dengue and dengue hemorrhagic fever. Lancet. 1998;352(9132):971-977.

https://doi.org/10.1016/s0140-6736(97)12483-7

2. Mairuhu AT, Wagenaar J, Brandjes DP and van Gorp EC. Dengue: An arthropod-borne disease of global importance. Eur J Clin Microbiol Infect Dis. 2004;23(6):425-433. https://doi.org/10.1007/s10096-004-1145-1

3. World Health Organization. Global Stratergy for Dengue Prevention and Control, 2012-2020, Geneva: WHO Press; 2012.

4. National Vector Borne Disease Control Programme. Dengue Cases and Deaths in the Country Since. NVBDCP; 2010.

5. Yacoub $S$, Wertheim $H$, Simmons $C P$, Screaton $G$ and Wills $B$. Cardiovascular manifestations of the emerging dengue pandemic. Nat Rev Cardiol. 2014;11:335-345.

https://doi.org/10.1038/nrcardio.2014.40

6. Kautner I, Robinson MJ and Kuhnle U. Dengue virus infection: Epidemiology, pathogenesis, clinical presentation, diagnosis, and prevention. J Pediatr 1997;131(4):516-524.

https://doi.org/10.1016/s0022-3476(97)70054-4

7. Nimmannitya S. Clinical spectrum and management of dengue haemorrhagic fever. Southeast Asian J Trop Med Public Health. 1987;18(3):392-397.

8. Solomon T, Dung NM, Vaughn DW, Kneen R, Thao LT, Raengsakulrach $B$, et al. Neurological manifestations of dengue infection. Lancet. 2000;355(9209):1053-1059.

https://doi.org/10.1016/s0140-6736(00)02036-5

9. Lawn SD, Tilley R, Lloyd G, Finlayson C, Tolley H, Newman P, et al. Dengue hemorrhagic fever with fulminant hepatic failure in an immigrant returningto Bangladesh. Clin Infect Dis. 2003;37(1):e1-e4. https://doi.org/10.1086/375601

10. Mahmod M, Darul ND, Mokhtar I, Nor NM, Anshar FM and Maskon $\mathrm{O}$. Atrial fibrillation as a complication of dengue hemorrhagic fever: Non-self-limiting manifestation. Int $\mathrm{J}$ Infect Dis. 2009;13(5):e316-e318. 
https://doi.org/10.1016/j.ijid.2009.01.017

11. Pothapregada S, Kamalakannan B and Thulasingam M. Clinical profile of atypical manifestations of dengue fever. Indian $\mathrm{J}$ Pediatr. 2016;83(6):493-9.

https://doi.org/10.1007/s12098-015-1942-9

12. Sreenivasa B, Manjunatha B and Nivil J. Bleeding manifestations in dengue and their correlation with the platelet count. Sri Lanka J Child Health. 2017;46(3):218-221.

https://doi.org/10.4038/sljch.v46i3.8320

13. Kakoti G, Dutta P and Das BR. Dengue encephalitis: An atypical manifestation of dengue fever in children. Int J Health Sci Res. 2016;6(4):1-7.

\section{Authors Contribution:}

SP - Concept and design of the study; BR-Interpretation of result and first draft of manuscript; AC - Review of literature and statistical analysis;

AA - Manuscript preparation; GD - Revision of manuscript.

Work attributed to:

Calcutta National Medical College, 32, Gorachand Road, Beniapukur - 700 014, Kolkata.

Orcid ID:

Dr. Sumita Pal - (1) https://orcid.org/0000-0001-9546-1173

Dr. Basudha Roy - (D) https://orcid.org/0000-0002-8241-7242

Dr. Amrit Chattopadyay - (D) https://orcid.org/0000-0003-3006-6003

Dr. Akhila Andra - (1) https://orcid.org/0000-0003-4203-7517

Dr. Gautam De - (1) https://orcid.org/0000-0002-7964-6765

Source of Funding: None, Conflicts of Interest: None. 